\title{
Link-Layer Software-Defined Addressing using Block Address Registration and Claiming (BARC)
}

This paper was downloaded from TechRxiv (https://www.techrxiv.org).

\section{LICENSE}

CC BY 4.0

SUBMISSION DATE / POSTED DATE

01-02-2022 / 03-02-2022

\section{CITATION}

Marks, Roger (2022): Link-Layer Software-Defined Addressing using Block Address Registration and Claiming (BARC). TechRxiv. Preprint. https://doi.org/10.36227/techrxiv.19105118.v1

$\mathrm{DOI}$ 


\title{
Link-Layer Software-Defined Addressing using Block Address Registration and Claiming (BARC)
}

\author{
Roger B. Marks, Fellow, IEEE \\ EthAirNet Associates \\ Denver, CO, USA \\ roger@ethair.net
}

\begin{abstract}
Link layer network technology is evolving to take advantage of dynamic software-defined addressing (SDA). IEEE 802 standards have begun to support such an evolution by providing interoperable assignment of local addresses. This paper explores address assignment using the Block Address Registration and Claiming (BARC) protocol, under consideration in the P802.1CQ standardization project. Application examples illustrate the use of BARC and its potential to support new link layer networking capabilities.

Index Terms - software-defined addressing (SDA), Block Address Registration and Claiming (BARC), flow-zone switching (FZS), IEEE 802.1CQ, local address assignment, link layer
\end{abstract}

\section{INTRODUCTION}

An IEEE 802 network delivers a link-layer frame to a destination primarily on the basis of an IEEE 802 address, generally known as a medium-access control (MAC) address, carried in the frame as a destination address (DA). Such addresses are specified in IEEE Std 802 [1]. A bit splits the IEEE 802 address space into globally unique and locallyunique addresses; another bit divides the space into unicast and multicast addresses. Sets of global addresses are assigned by the IEEE Registration Authority [2] to applicants, who are responsible to assign unicast addresses within the set uniquely and permanently to hardware. The IEEE Registration Authority manages the address space with the goal of ensuring that sufficient addresses are available for at least 100 years since the opening of the registry in around 1980. Nevertheless, the proliferation of devices, many of which consume multiple addresses, points toward the inevitable exhaustion of the global address inventory.

Current developments emphasize the need for dynamically assigned addresses. For example, virtual machines in data centers may be assigned addresses at startup, and mobile devices increasingly seek to use temporary addresses in order to avoid a tracking trail. These trends have led to further exploitation of the local IEEE 802 address space. An IEEE 802 network operates at least as well with local as with global addresses, as long as they are unique within that network; in fact, as discussed below, networks have the potential to work more efficiently with local addresses. In 2017, IEEE Std 802 was amended by IEEE Std 802c [3], which provides a structure of the local address space promoting unique assignment.

While unicast addresses designate a single network interface, such as an end station or bridge port, multicast addresses are assigned to identify groups of receivers and, accordingly, are inherently dynamic. In some cases, a multicast address indicates a protocol that all interested devices receive. In other cases, a multimedia stream is sent to a group of listeners, with the group dynamically configurable, as illustrated in Section V-E.

IEEE Project P802.1CQ (on "Multicast and Local Address Assignment") [4], organized within the Time-Sensitive Networking (TSN) Task Group of the IEEE 802.1 Working Group, is taking steps to further the exploitation of the local MAC address space, and to aid in the effective use of multicast addresses, by developing a standard protocol for address assignment. The current draft (P802.1CQ/D0.7) details the "Block Address Registration and Claiming" protocol (BARC). BARC allows a claimant to communicate a Discover message indicating an interest in attaining one or more address assignments. A BARC registrar on the network can respond with an assignment from a managed address pool. In the absence of a BARC registrar, the BARC claimant proceeds to self-assignment. BARC claimant peers respond, defending their own previously self-assigned addresses, and the BARC claimant self-assigns its address so as to avoid defended addresses. The draft also describes a BARC advisor entity that can propose an address claim and recommend a registrar in response to an Inquiry message.

BARC address assignments are always made in terms of a specified address block (AB). Each address block includes both unicast and multicast subblocks, identical except for a single bit. Each subblock includes a contiguous set of addresses. Claimable ABs (CABs) are specified with 1, 16, 256, or 4096 addresses per subblock. Registrable ABs (RABs) may be of the same dimensions or may be aggregated into even larger ABs. Each address block is uniquely identified by a 48bit identifier. The identifier of a $\mathrm{CAB}$ is a multicast address that does not belong to the $\mathrm{CAB}$.

This paper details the main principles of BARC and its operation. It also explores possible applications of BARC, explaining why software-defined address assignment, particularly with block assignment, can offer new opportunities for link-layer functionality.

An earlier brief overview of IEEE Project P802.1CQ [5] summarized the project prior to the introduction of BARC.

(C) 2021 IEEE. Personal use of this material is permitted. Permission from IEEE must be obtained for all other uses, in any current or future media, including reprinting/republishing this material for advertising or promotional purposes, creating new collective works, for resale or redistribution to servers or lists, or reuse of any copyrighted component of this work in other works. 


\section{ADDRESS FORMAT PER IEEE STD 802}

Table I illustrates a 48-bit IEEE 802 data frame address, shown as six octets (one per row) and dividing each octet into nibbles (two per row).

TABLE I

LAYER 2 ADDRESS

\begin{tabular}{|l|c|c|}
\hline byte & more significant nibble & less significant nibble \\
\hline B0 (MSB) & N0 = rijk & N1 = zyxm \\
\hline B1 & N2 & N3 \\
\hline B2 & N4 & N5 \\
\hline B3 & N6 & N7 \\
\hline B4 & N8 & N9 \\
\hline B5 (LSB) & N10 & N11 \\
\hline
\end{tabular}

The least significant bits of nibble N0 hold special significance. In order, from least significant:

- the $\mathrm{m}$ or "I/G" bit is set to 0 for an individual (unicast) address and to 1 for a group (multicast) address;

- the $x$ or "U/L" bit is set to 0 for a universal (global) address and to 1 for a local address;

- $y$ and $z$ are not specified for global addresses. For local addresses, however, IEEE Std 802c [3] introduced the optional Structured Local Address Plan (SLAP) that divides the local address space into four quadrants on the basis of those two bits. In particular, an address with $x=y=z=1$ is a local address in the SLAP quadrant specified for a Standard Assigned Identifier (SAI). Ref. [3] reserves specification of the use of the SAI quadrant for SLAP address assignments to IEEE P802.1CQ [4].

\section{BARC ADDRESS FORMAT}

BARC has been developed for the SLAP SAI quadrant only. It encodes additional information in the bits of nibble NO. Namely:

- when $r=1$, the address is a Registrable Address (RA) in a Registrable Address Block (RAB);

- when $r=0$ and $i=1$, the address is a Claimable Address (CA) in a Claimable Address Block (CAB) of size $j k$;

- when $r=0, i=0$, and $m=1$, the address is a Claimable Address Block Address (CABA) that identifies the associated $\mathrm{CAB}$ with $i=1$;

- when $r=0, i=0$, and $m=0$, the address is a temporary unicast address (TUA) for use in BARC messaging only.

The key features of the BARC address formatting scheme are:

- each $C A B$ and $R A B$ includes contiguous unicast and multicast subblocks, identical except for the $m$ bit;

- each $\mathrm{CAB}$ is disjoint from each other $\mathrm{CAB}$ and from all RABs;

- each CAB is identified by a CABA that is a multicast address used only for BARC messaging. This feature allows announcements regarding a particular $\mathrm{CAB}$ to be received by only devices interested in exactly that $\mathrm{CAB}$, as explained in Section IV-A.
Fig. 1 illustrates the $\mathrm{CA}, \mathrm{CAB}$, and $\mathrm{CABA}$ structure for the four $\mathrm{CAB}$ sizes. Nibble $\mathrm{N} 2$ of each $\mathrm{CA}$ is set to 0 , allowing for the coexistence of alternative SAI address formats that may use alternate values. The $\mathrm{CAB}$ size is specified to allow CABs of four different sizes to coexist. As shown, the $i j$ least significant nibbles of the $\mathrm{CAB}$ are "wildcard" nibbles such that all values of those nibbles are included in the CAB. The $\mathrm{CAB}$ structure includes:

- $\approx 7 \cdot 10^{10} j k=0$ CABs with 1 address per subblock;

- $\approx 4 \cdot 10^{9} j k=1$ CABs with 16 addresses per subblock;

- $\approx 3 \cdot 10^{8} j k=2 \mathrm{CABs}$ with 256 addresses per subblock;

- $\approx 2 \cdot 10^{7} j k=3$ CABs with 4096 addresses per subblock.

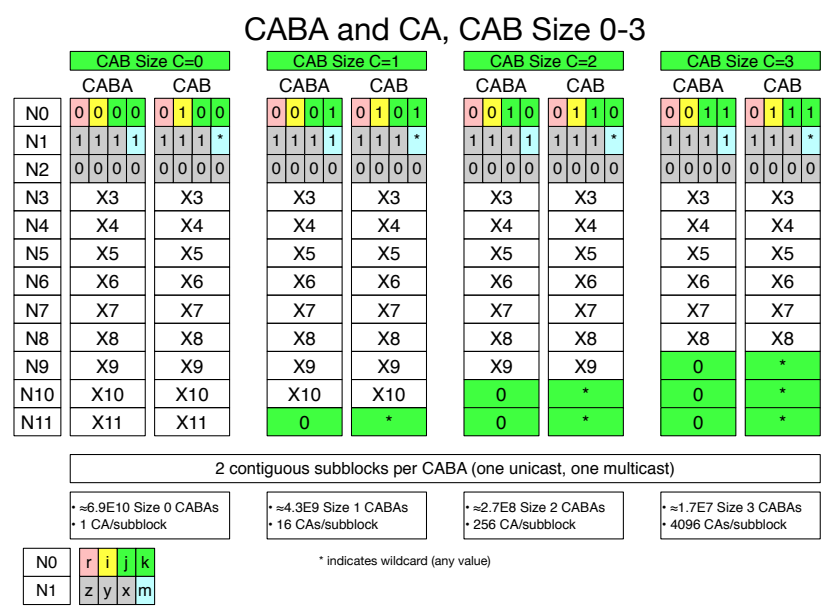

Fig. 1. $\mathrm{CA}, \mathrm{CAB}$, and $\mathrm{CABA}$ structure

This provides a vast number of CAs for a single local area network (LAN), so that wastage, by assignment of unused addresses, is not a critical factor in many cases. BARC anticipates that many, or even most, assigned addresses will be unused. For example, some claimants will require only unicast, or only multicast, addresses. Furthermore, BARC specifies that each 48-bit address assignment includes all of the 64bit addresses that can be created by appending an arbitrary 16-bit suffix; for an example of use, see Section V-D. Many of these addresses will also remain unused.

BARC specifies a similar structure for RABs, although with some differences. All values of $\mathrm{N} 2$ are allowed in a RAB, considering that the address assignment is under the control of a registrar that can be configured to limit assignments to prevent conflicts with alternate protocols. A RAB is identified by a 48-bit RAB Identifier (RABI). RABs can be aggregated into larger blocks for assignment to registrars; from that assigned inventory, the registrar creates assignments for claimants.

\section{BARC PROTOCOL}

The BARC protocol operates with the exchange of fixedsize BARC protocol data units (PDUs). A BARC PDU contains a 48-bit BARC identifier, a 48-bit BARC address, a 48-bit information parameter, and a state parameter. The BARC PDU is 40 bytes, small enough to fit within the data payload of a 
minimum size (64 byte) Ethernet frame, even if a VLAN tag is added.

Various BARC messaging sequences are possible. Basic sequences include:

\section{A. CABA claim without registrar}

The claiming process without registrar is indicated schematically in Fig. 2. A claimant begins by selecting a $\mathrm{CAB}$ to claim, based on the number of addresses needed and possibly on its preferred values of nibbles N3-N11. It creates a BARC PDU specifying the associated CABA (here $\mathrm{CABA}_{0}$ ) in the Discover state. It transmits the PDU to the network in a frame in which the source address may be a TUA, used only for BARC messaging, and using $\mathrm{CABA}_{0}$ as the DA. Another claimant holding the $\mathrm{CABA}_{0}$ assignment responds with a unicast BARC PDU to defend the claim; upon receipt of the defending PDU, the claimant drops that claim and begins afresh with an alternate one $\left(\mathrm{CABA}_{1}\right)$. Since $\mathrm{CABA}_{n}$ is a multicast address that is uniquely associated with the claimed $\mathrm{CAB}$, only a claimant holding exactly that $\mathrm{CAB}$ listens for messages at that DA. In a typical network, a claimant will not be interrupted by claims made to non-conflicting CABAs because it will not listen for them. In Fig. 2, the claim for $\mathrm{CABA}_{1}$ is heard by no other claimant. When a timer expires without a response, the claimant sets $\mathrm{CABA}_{1}$ into Claimed state and announces that fact to $\mathrm{CABA}_{1}$.

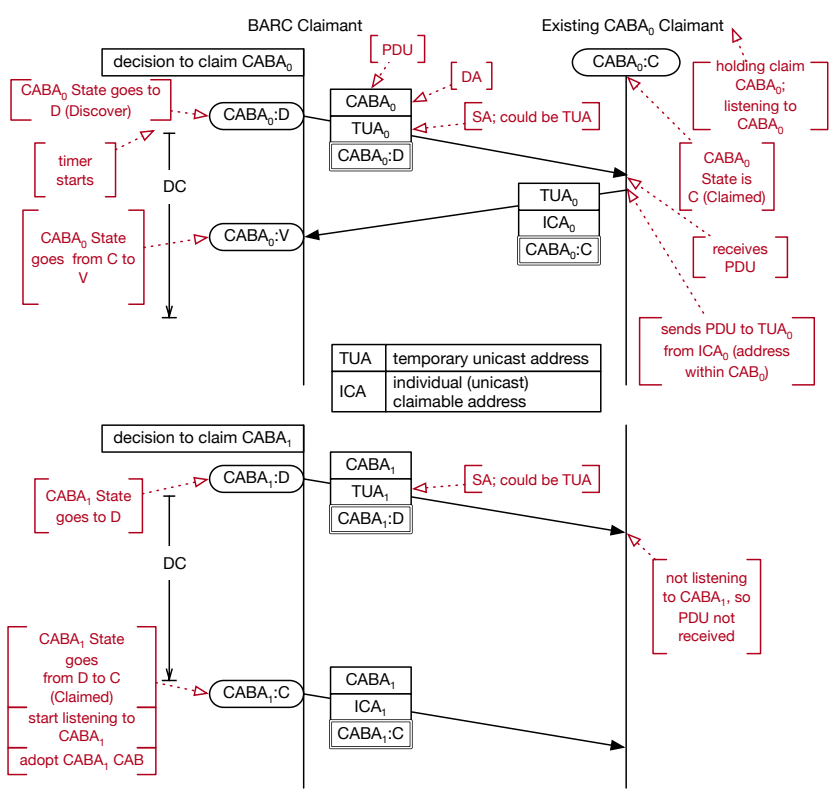

Fig. 2. CABA claim without registrar

\section{B. CABA claim with registrar}

The claiming process with registrar is indicated schematically in Fig. 3. A claimant begins without knowledge of the availability of a registrar or advisor and therefore transmits a CABA Discover PDU to a CABA, just as in the prior example. In this case, however, a registrar on the network hears that PDU; such a registrar listens for messages sent to any CABA.
The registrar responds with a unicast Offer message specifying a RABI that identifies a RAB offered to the claimant. If the claimant accepts the offer, a message sequence (not shown) completes the registration of the RAB and its assignment to the claimant. The claimant may select among multiple offers received from multiple registrars and therefore awaits a timeout before choosing one. In the example, the Offer message includes a defense of the original CABA claim, so the claimant discontinues that claim.

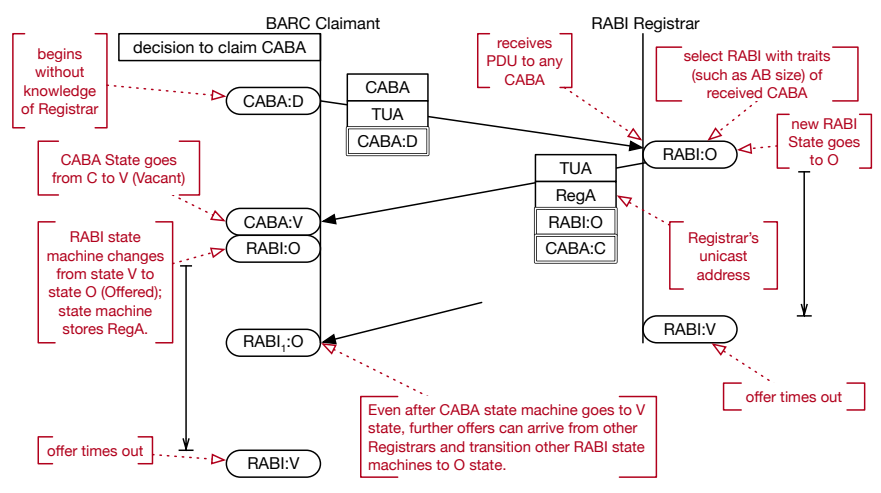

Fig. 3. CABA claim with registrar

\section{Claimant inquiry and advisor proposal}

A claimant that anticipates a registrar or advisor on the network may send an Inquiry message to the anticipated address. If the address is unknown but the registrar or advisor is anticipated directly at the claimant port, without bridging, the DA may be the generic nearest customer bridge (NCB) address specified as non-forwarding by bridges per IEEE Std 802.1Q [6]. An advisor may respond with a BARC message proposing a CABA, or a set of proposed RABIs, and an address of a recommended registrar for a followup Inquiry. A registrar may also respond to an Inquiry with an Offer, which may lead to a registration sequence.

\section{IMPLICATIONS AND APPLICATIONS}

The IEEE 802 global address provides one designated informational bit (the $m$ bit). The purpose of the remainder is only to provide uniqueness. Some additional information can be gleaned by identifying the global IEEE RA assignment set of the block, but this is of minimal use during operation since it represents a factory assignment that does not foresee the network.

A core application of local address assignment is the replacement of permanent global addresses to defer the exhaustion of the inventory and potentially to reduce the complexity of differentiation at the factory. However, the nature of the BARC assignment provides opportunities to add more value to the address. BARC registrar and advisor entities can direct the assignment of local address blocks that may be coded to contain semantic information valuable in the operation of the network. BARC may therefore be used to provide dynamic software-defined addressing (SDA) that provides addresses that are functional as well as unique. Some examples follow. 


\section{A. Location-based addressing}

A key use of SDA is for location-based addressing, as exemplified in Fig. 4, which illustrates two access switches (A and $\mathbf{B}$ ) connected to a LAN. Each switch connects four servers $(\mathbf{x}, \mathbf{y}, \mathbf{Z}, \mathbf{w})$ via ports with local port identifiers $(\mathbf{1 , 2 , 3 , 4})$. The servers support virtual machines (a,b,c). Virtual machine (VM) $\mathbf{a}$ at server $\mathbf{x}$ of access switch $\mathbf{A}$ is assigned a RAB indicating $\mathbf{a}$ in nibbles $\mathrm{N} 10$ and N11, $\mathbf{x}$ in nibble N9, and $\mathbf{A}$ in nibbles N6N8. Nibbles N0-N5 are configured to form a semantic prefix that can be decoded to determine the structure of the address so it can be parsed. In the LAN, a frame whose DA is a unicast address in this RAB is examined and parsed according to the semantic prefix. Accordingly, the LAN identifies N6$\mathrm{N} 8$ as the destination field, looks up N6-N8 in a forwarding table, and forwards the frame toward access switch A. The access switch, reading the semantic prefix, identifies N9 as the destination field, looks up N9 in a forwarding table, identifies the associated port, and forwards the frame via that port toward to server $\mathbf{x}$. Server $\mathbf{x}$, reading the semantic prefix, identifies N10-N11 as the destination field and forwards the frame to the VM a associated with N10-N11. This method uses a form of route aggregation, obviating the need for the switch element to learn entire MAC addresses or hold large forwarding tables, and forwarding performance may consequently be improved compared to conventional bridge forwarding.

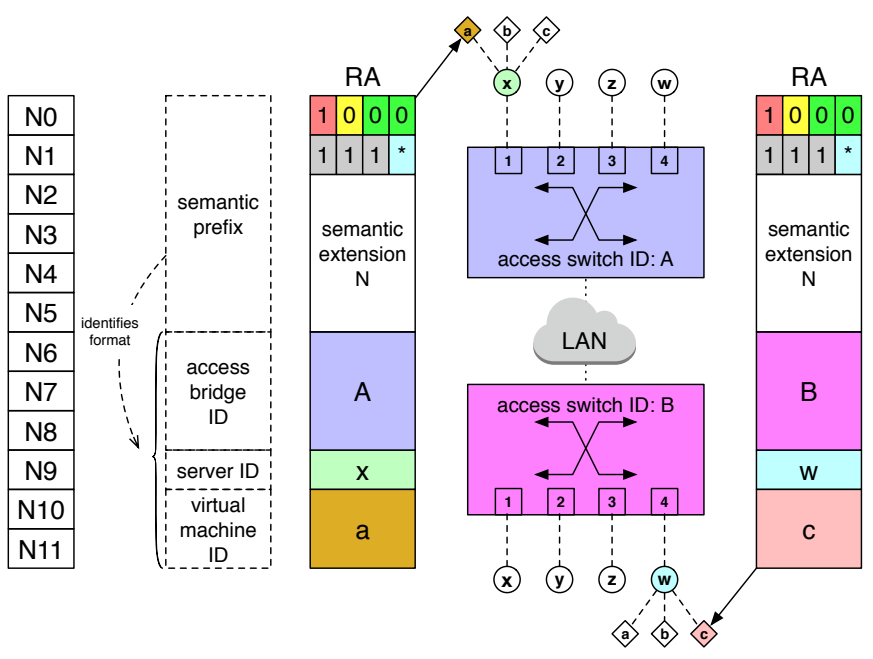

Fig. 4. Location-based address assignments

These RAB assignments could be provided directly by Discover claims to a central registrar. However, that registrar would not know the topology, so the assigned RABs would not be meaningful until the forwarding tables were populated. This could be done by, for example, flooding and learning. Alternatively, the Inquiry process provides the means to improve this situation, because an Inquiry can be directed to the nearest neighbor using the non-forwarding NCB address. For example, an Inquiry from $\mathbf{x}$ to the NCB address is received by $\mathbf{A}$ alone. If $\mathbf{A}$ is enabled as an advisor, it can propose to $\mathbf{x}$ a RAB that is consistent with its own and also includes, in nibble N9, the appropriate server ID for $\mathbf{x}$. The simplest way for $\mathbf{A}$ to identify $\mathbf{x}$ is by the local port identifier $\mathbf{1}$; in other words, using the local port identifier ("1") as the server ID (N9). The additional benefit of such an assignment is that A subsequently forwards to $\mathbf{x}$ statelessly, with no forwarding table required, since the forwarding port is recorded explicitly in the frame. Such an address assignment process can be extended to other levels of the network, based on exchange of Inquiry messages and responding proposals. The claimant receiving the proposal is provided with not only with a suitable proposed RAB but also the address of a registrar at which to request assignment. Is it also possible for the neighbor to respond directly with a RAB Offer, rather than a proposal referring the claimant to a registrar, at the cost of implementing distributed registrar functionality throughout. Note that, even with distributed registrars, the Inquiry rather than the Discover process is necessary since the multicast Discover message would result in multiple responses for the claimant to choose among.

\section{B. Flow-zone switching (FZS)}

A comprehensive version of SDA is explored in the flowzone switching (FZS) method [8]. FZS structures frames to include not only the topological location but also flow identifiers that can be used to direct forwarding and Quality of Service (QoS) treatment. The primary topology discussed in [8] is a generalized Clos network, as commonly used in data centers, that can scale up to $2^{24}$ servers, with $256 \mathrm{VMs}$ per server and 256 flows per VM. The address formats considered in [8] support stateless forwarding and are compatible with BARC addressing. They use a semantic prefix to identify the topological level of the address and structure the remainder of the address, in accordance with that semantic prefix, to designate a specific location as well as flow identification. An example illustrates how this can allow a source to specify a preferred route among several routes, all leading to the location specified within the DA, that are nominally equal in cost. A numerical study separates TCP data frames from pure TCP acknowledgement frames in order to improve the latency and reliability of the smaller acknowledgement frames.

In a hyperscale data center, the assignment of suitable address blocks is a nontrivial problem. The automated address assignment detailed in [8] is not compatible with BARC. However, alternatives versions that make use of the BARC Inquiry process are possible, following the principles described in the prior subsection.

\section{IEEE 802.11 wireless LANs}

An important concern with global addresses for wireless LANs (WLANs), based on IEEE Std 802.11 [9], is that they endanger the privacy of the mobile user when a permanent addresses is indiscriminately shared over the air and to access points. Recently, this problem has been mitigated by replacing the global address with a randomly-generated local address. This resolves the privacy issue to a large degree, but it has some negative side effects. For example, address collisions, though unlikely, are possible, and no collision protection 
is available except in special cases; e.g., when the MAC address is incorporated into a higher-layer address that is checked for duplicates, as might occur in some cases of IPv6 Stateless Address Autoconfiguration. Also, some applications dependent on MAC addresses for identification need to be updated to account for temporary addresses.

SDA can resolve the privacy issues, avoiding side effects, and also provide new opportunities as well. Local addresses inherently interfere with tracking, even if they are not continually re-randomized. A network-designated address block assignment could be customized based on, for example, a prior assignment on the same network; this would allow continuity on the network but limit the information required to track a device as it roamed across networks. Furthermore, the network can assign addresses based on location in the network topology; in particular, like the earlier example, the address block root could be assigned to the access point at which the association is initiated. This would allow the network to forward frames to the access point based on the root, without learning the MAC address. It some cases, the address block root could be assigned to an access switch behind an access point, in order to accommodate roaming among access points without an address change.

An issue with WLAN is to ensure that address assignments are made prior to association, since the address cannot be changed within the context of that association. Another issue is that the WLAN network is not optimal for multicast claiming. Fortunately, IEEE Std 802.11 [9] provides pre-association communications methods, including Access Network Query Protocol (ANQP), providing a potential platform for the ANQP server to provide a RABI or set of RABIs to the station before authentication proceeds.

\section{Bridging of 64-bit LANs}

As previous noted, BARC assigns, along with each 48bit address, all of the 64-bit addresses formed by extending it by 16 bits. To illustrate an application of this approach, consider that IEEE 802 networking can operate with 48 or 64 bit addresses, but these are never mixed. IEEE 802 networks operating with 64-bit addresses include those based on IEEE Std 802.15.4 [10]. These networks do not make use of IEEE 802 bridging since IEEE 802 bridges are specified only for 48bit addresses. Consider, however, Fig. 5, which illustrates (in three different colors) three 64-bit LANs, as might be deployed in separate areas of a facility.

Generally speaking, bridging should enable the devices in these LANs to communicate with each other. In the absence of functional link-layer bridging, Layer 3 routing could be used, but this would require significant packet overhead and extra functionality in the end stations. In Fig. 5, each network includes a bridge interface to a 48-bit bridged LAN. In the example, each of the bridge interfaces has been allocated a 48-bit address, which includes an assignment of all of the extended 64-bit addresses. The stations of the 64-bit LAN are assigned BARC addresses based on the root address of the bridge interface; in some cases, the 16-bit extension could

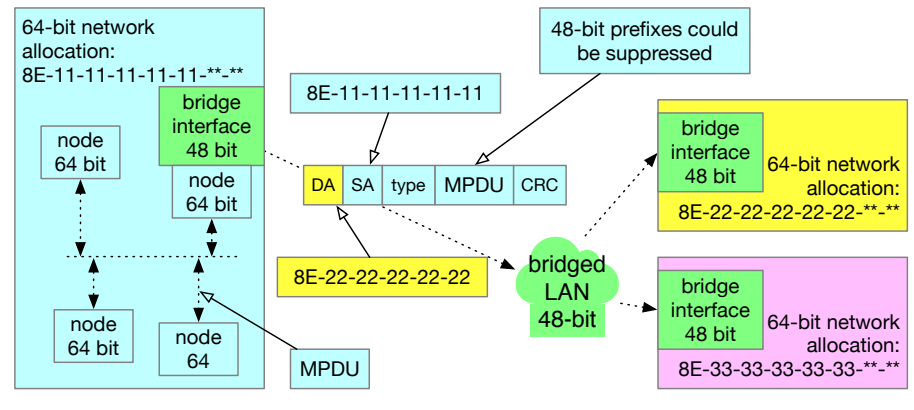

Fig. 5. 48-bit bridging of 64-bit LANs

serve as a short address within the LAN. In the 64-bit LAN, any frame addressed to a device outside that LAN is sent to the bridge interface, which incorporates the MAC Protocol Data Unit (MPDU) as the payload a 48-bit frame whose destination and source addresses, respectively, are the 48-bit roots of the 64-bit addresses. The bridged LAN, operating in a conventional way and using only one forwarding table entry per LAN, forwards the frame to the correct bridge interface, which extracts the MPDU payload for delivery to the destination. In this way, the bridged LAN, in effect, bridges all of the 64-bit LANs into a single transparent LAN. Notably, no address translation tables are needed at the bridge interface. Note also that, in this description, the overhead is unnecessarily large if the 48-bit root of each 64-bit address is carried twice in each packet. However, with agreement on a standard header-suppressed format, such duplication would be eliminated, saving 12 bytes per frame and with the potential to carry the 64-bit frame in 48-bit form with no added overhead.

Other modes of operation are also possible. For example, each 64-bit LAN could be assigned an $\mathrm{AB}$ large enough to accommodate all its devices. In this case, each 48-bit address could map to a single 64-bit address; for example, by adding 16 bits of 0 . The bridge could operate conventionally but would require a large forwarding table, unless it were configured to forward based on the address root.

\section{E. Multicast streams}

Multicast address are sometimes assigned generically to a protocol; all interested devices know to listen for frames there, and global assignment may be useful. Other scenarios, however, suggest the need for dynamically assigned local multicast addresses. As an example, consider a source that transmits one or more audiovisual streams. Each of several streams may have a different set of listeners; these sets can be distinguished by multicast DA. In such a scenario, multicast addresses are logically assigned not to protocols but to sources. An example of such use is included in the Audio Video Transport Protocol of [7], which specifies the MAC Address Acquisition Protocol (MAAP) to prevent duplication of multicast addresses claimed by devices for use as stream DAs. BARC has some advantages over MAAP; for example, it supports registrar assignment, and it supports devices lacking an initial unicast address with which to initiate a claim. Also, since it assigns both unicast 
and multicast addresses simultaneously, it is applicable to streaming sources that require an assigned source address block. Additional advantage could be drawn from the fact that the unicast and multicast assignments are identical except for the $m$ bit.

\section{FUTURE DIRECTIONS}

While SDA offers the opportunity for increased switching efficiency, that opportunity is realized only when bridges are enabled to parse addresses, interpret the address format, extract relevant location and flow information, and consequentially make an appropriate forwarding decision. IEEE Std 802.1Q already incorporates, in addition to address learning and VLAN processing, some programmable forwarding techniques, such as Multiple MAC Registration Protocol (MMRP), which allows declaration of specific forwarding for address ranges. Improvements based on BARC assignments are possible and could be considered for future standardization. Messaging to inform bridges of custom address formats could prove useful.

Security considerations are another area for future study. In some regards, BARC may reduce some threats; for example, while global addresses as well as local addresses can be spoofed, BARC might in some cases increase the challenges for the spoofer since the network could limit allowed source addresses based on the address assignment policy. MMRP is known to be risky in the case of unicast address declarations and could be assessed in a BARC environment. Many other threat scenarios could and should be studied; this could include, for example, claimants that retain a claim beyond its lifetime or after a mobility event, and registrars operating outside administrative authority. Authentication and message encryption deserve study. Also, while the current BARC specification include mechanisms to manage disruptions due to network merging, the issue should be modeled in detail.

\section{CONCLUSION}

Dynamic software-defined addressing, using BARC address assignment, offers the opportunity to efficiently introduce new functionality into link-layer networks using standardized frame formats with customized content. Such addressing can support efficient routing and forwarding methodologies with minimized forwarding tables and lookups, or even without such tables, and with minimal frame overhead.

\section{ACKNOWLEDGMENTS}

The author appreciates insights provided by participants in IEEE 802 Project P802.1CQ, particularly by Antonio de la Oliva, Lily Lv, Norm Finn, Don Pannell, Mick Seaman, and Pat Thaler. He also appreciates discussions with Elisa Rojas and Sergio Gonzalez-Diaz concerning flow-zone switching, and the comments of anonymous reviewers of the manuscript.

\section{ABBREVIATIONS}

$\begin{array}{ll}\text { AB } & \text { address block } \\ \text { ANQP } & \text { Access Network Query Protocol } \\ \text { BARC } & \text { Block Address Registration and Claiming } \\ \text { CA } & \text { claimable address } \\ \text { CAB } & \text { claimable address block } \\ \text { DA } & \text { destination address } \\ \text { FZS } & \text { flow-zone switching } \\ \text { ID } & \text { identifier } \\ \text { I/G } & \text { individual/group (bit) } \\ \text { LAN } & \text { local area network } \\ \text { LSB } & \text { least significant byte } \\ \text { MAC } & \text { medium-access control } \\ \text { MMRP } & \text { medium-access control } \\ \text { MSB } & \text { most significant byte } \\ \text { NCB } & \text { nearest customer bridge } \\ \text { PDU } & \text { protocol data unit } \\ \text { RA } & \text { registrable address } \\ \text { RAB } & \text { registrable address block } \\ \text { SAI } & \text { Standard Assigned Identifier } \\ \text { SDA } & \text { software-defined addressing } \\ \text { SLAP } & \text { Structured Local Address Plan } \\ \text { TUA } & \text { temporary unicast address } \\ \text { U/L } & \text { universal/local (bit) } \\ \text { VLAN } & \text { virtual local area network } \\ \text { VM } & \text { virtual machine } \\ \text { WLAN } & \text { wireless local area network }\end{array}$

\section{REFERENCES}

[1] IEEE Standards Association, "IEEE Standard for Local and Metropolitan Area Networks: Overview and Architecture,” IEEE Std 802-2014 https://ieeexplore.ieee.org/document/6847097.

[2] IEEE Standards Association Registration Authority, "Guidelines for use of Extended Unique Identifier (EUI), Organizationally Unique Identifier (OUI), and Company ID (CID)," 3 August 2017 https://standards.iee.org/content/dam/ieeestandards/standards/web/documents/tutorials/eui.pdf.

[3] IEEE Standards Association, "IEEE Standard for Local and Metropolitan Area Networks: Overview and Architecture - Amendment 2: Local Medium Access Control (MAC) Address Usage," IEEE Std 802c-2017 https://ieeexplore.ieee.org/document/8016709.

[4] IEEE Standards Project P802.1CQ, "Multicast and local address assignment" https://1. ieee802.org/tsn/802-1cq.

[5] Roger B. Marks, "P802.1CQ: Multicast and local address assignment," IEEE Communications Standards Magazine: Volume 4, Issue 3, September 2020 https://ieeexplore.ieee.org/document/9424558.

[6] IEEE Standards Association, "IEEE Standard for Local and Metropolitan Area Network-Bridges and Bridged Networks," IEEE Std 802.1Q-2018 https://ieeexplore.ieee.org/document/8403927.

[7] IEEE Standards Association, "IEEE Standard for a Transport Protocol for Time-Sensitive Applications in Bridged Local Area Networks," IEEE Std 1722-2016 https://ieeexplore.ieee.org/document/7782716.

[8] Sergio Gonzalez-Diaz, Roger Marks, Elisa Rojas, Antonio de la Oliva, and Robert Gazda, "Stateless flow-zone switching using software-defined addressing," IEEE Access: 6 May 2021. https://ieeexplore.ieee.org/document/9424558.

[9] IEEE Standards Association, "IEEE Standard for Information Technology-Telecommunications and Information Exchange between Systems - Local and Metropolitan Area Networks-Specific Requirements - Part 11: Wireless LAN Medium Access Control (MAC) and Physical Layer (PHY) Specifications," IEEE Std 802.11-2020 https://ieeexplore.ieee.org/document/9363693.

[10] IEEE Standards Association, "IEEE Standard for LowRate Wireless Networks," IEEE Std 802.15.4-2020 https://ieeexplore.ieee.org/document/9144691. 